\title{
ORIGINAL
}

\section{PRÁCTICAS PREVENTIVAS DE LOS HABITANTES MAYORES DE 25 AÑOS EN MONTERREY Y SU ZONA METROPOLITANA (MÉXICO) (*)}

ME Garza-Elizondo, (1,2), E Villarreal-Ríos (3), AM Salinas-Martínez (1,4) y GM Núñez-Rocha (1) (1) Unidad de Investigación Epidemiológica y en Servicios de Salud. Instituto Mexicano del Seguro Social. Nuevo León. México.

(2) Facultad de Enfermería. Universidad Autónoma de Nuevo León. México.

(3) Unidad de Investigación Epidemiológica y en Servicios de Salud. Instituto Mexicano del Seguro Social. Querétaro. México.

(4) Departamento de Medicina Preventiva. Facultad de Medicina. Universidad Autónoma de Nuevo León. México.

\section{RESUMEN}

Fundamento: Los padecimientos crónicos y degenerativos son las primeras causas de morbi-mortalidad en México, por lo que el Sector Salud ha implementado acciones de prevención y detección oportuna. El uso de los servicios de salud es una conducta dinámica de la población. Para que la gente utilice estas acciones de prevención es necesario que se reduzcan barreras de acceso. Así, el objetivo del estudio fue determinar la utilización de los servicios de detección de diabetes mellitus, hipertensión arterial, cáncer cérvicouterino y mamario y aplicación del toxoide tetánico diftérico.

Métodos: El tamaño de la muestra fue de 254 personas de 25 años y más, que habitaban en Monterrey y su zona metropolitana. En el uso de acciones preventivas se consideró a las personas que las habían usado el año anterior. El análisis consistió en estadística descriptiva y análisis bivariado.

Resultados: Más del 60\% de la población correspondió al sexo femenino, la edad promedio fue de 42,3 años \pm 14 años y tres cuartas partes de la población contó con seguridad social. Un 37\% mencionó haberse realizado la detección de diabetes y un $44,5 \%$ de hipertensión, mientras que le aplicaron el toxoide tetánico diftérico a un $31,1 \%$. En las detecciones propias de la mujer, a un $34,3 \%$ de la couterino en tanto que a un $29,5 \%$, la de cáncer mamario. No hubo relación entre uso de acciones con antecedentes familiares y percepción de importancia de la detección.

Conclusiones: El uso de las acciones preventivas está por debajo de algunos estándares internacionales. Es necesaria la búsqueda de personas expuestas al riesgo para detectar oportunamente cualquier padecimiento crónico.

Palabras clave: Prevención. Inmunización. Hipertensión. Diabetes mellitus. Neoplasmas del cuello uterino. Neoplasmas de la mama.

Correspondencia:

ME Garza-Elizondo

Morelos 133 ote. $5^{\circ}$ Piso

Col. Centro. CP 64000. Monterrey

NL México

Correo electrónico: garzaeme@aol.com

\section{ABSTRACT}

\section{Preventive Practices of the Inhabitants of Monterrey, México}

Background: Chronic and degenerative disorders are the leading causes of morbidity-mortality in Mexico, as a result of which the Health Sector has implemented preventive and suitable detection measures. The use of the health services is a dynamic behavior on the part of the population. In order for people to use these preventive measures, the barriers to accessing these services must be lessened. Hence, the objective of this study was that of ascertaining the use of the services for the detection of diabetes mellitus, high blood pressure, cervical-uterine and breast cancer and tetanus and diphtheria toxoide vaccinations.

Methods: The sample size was that of 254 individuals age 25 and over living in Monterrey or in the greater Monterrey metropoli$\tan$ area. Those having employed preventive measures during the year immediately prior to the study were taken into account with regard to the use of preventive measures. The analysis consisted of descriptive statistics and bivariate analysis.

Results: Over $60 \%$ of the population was female, the average age being $42,3+14$ years of age, three fourths of the population being on the social security rolls. A total $37 \%$ mentioned having undergone the diabetes test, and 44,5 the test for high blood pressure, while $31,1 \%$ had been vaccinated with the tetanus and diphtheria toxoide. Regarding specifically female checkups, $34,3 \%$ of all females had undergone the corresponding cervical-uterine cancer test, $29,5 \%$ having been screened for breast cancer. No relationship was found to exist between the use of measures and family histories and the perception of the importance of the checks.

Conclusions: The use of preventive measures fall below some international standards. Individuals exposed to the risk must be sought in order to fittingly detect any chronic disorder.

Key words: Prevention. Immunization. Diabetes mellitus. Hypertension. Cervix neoplasms. Breast neoplasms.

(*) Proyecto con apoyo financiero por parte del Fondo para el Fomento de la Investigación Médica, del Instituto Mexicano del Seguro Social; con el registro Número FP2001/111 


\section{INTRODUCCIÓN}

Uno de los retos que enfrenta el Sector Salud Mexicano (SSM) es la transición epidemiológica caracterizada por el traslape de enfermedades infecciosas y crónicas ${ }^{1}$. Estas últimas ocupan las primeras causas de morbilidad y mortalidad, ejemplo de ello son la diabetes mellitus, las enfermedades hipertensivas y el cáncer cérvicouterino, que durante el año 2000 fueron las causas más frecuentes de muerte en el país ${ }^{2}$. Lo anterior hace patente la necesidad de que la población expuesta al riesgo realice en los servicios de salud acciones encaminadas a detectar oportunamente estos padecimientos.

El SSM ha implementado una serie de estrategias en el ámbito nacional encaminadas a mejorar los niveles de bienestar de los mexicanos $^{3}$, entre las que se encuentran la prevención y control de cáncer cérvicouterino $^{4,5}$ y mamario ${ }^{5,6}$ la hipertensión arterial5,7 y la diabetes mellitus ${ }^{5,8}$, todas ellas derivadas de las Normas Oficiales Mexicanas (NOM). Las NOM para la detección de diabetes mellitus (DDM $)^{9}$ recomiendan una glucemia capilar cada dos años a partir de los veinte y una vez al año después de los 64 años de edad. Además, una glucemia sérica cada tres años en caso de que la capilar sea >de $110 \mathrm{mg} / \mathrm{dl}$ y en ausencia de factores de riesgo. Mientras que Estados Unidos recomienda su realización anual, tanto por el Programa Practicando la Prevención ${ }^{10}$ como por el Grupo de Expertos en Servicios Preventivos $^{11}$.

Las NOM para la detección de hipertensión arterial (DHTA) ${ }^{12}$ proponen la toma de la presión arterial cada dos años a partir de los veinte y cada seis meses después de los 64 años. En tanto que en España se recomienda cada cuatro o cinco años entre los 14 y 40 años y después cada dos años ${ }^{13}$, en Estados Unidos cada dos años para población norteamericana ${ }^{10,11}$. En lo que respecta a la vacunación en adultos se sugiere el toxoide tetánico-diftérico, aplicado en dos dosis y un refuerzo ${ }^{14-16}$. Dicha vacuna se encuentra entre las inmunizaciones recomendadas para la población en Estados Unidos ${ }^{10}$.

Entre las acciones preventivas propias de la mujer, las NOM sugieren la citología cérvicouterina cada tres años después de dos resultados negativos a partir de los 25 años para la detección de cáncer cérvicouterino (DCACU), mientras que en mujeres con resultados positivos a procesos inflamatorios inespecíficos se establece cada año hasta dos citologías negativas ${ }^{17}$. Para mujeres españolas se propone cada cinco años después de dos resultados negativos hasta los 65 años y con vida sexual activa; en mujeres mayores de esta edad y ante dos citologías anuales negativas, ya no más intervenciones ${ }^{18}$. En tanto que para las mujeres norteamericanas se recomienda cada tres años al inicio de la vida sexual o a los 21 años de edad; después de los 65 con resultado negativo, no más intervenciones ${ }^{10,11}$. En el caso de la detección de cáncer mamario (DCAMA) está legislado que se realice mediante la autoexploración mamaria a partir de la menarquia, además del examen físico anual de mamas a partir de los 25 años y una mamografía bienal, en mujeres con riesgo de los 40 años en adelante ${ }^{17}$. En España se recomienda el examen físico anual de mamas en caso de no tener disponible el recurso de la mamografía, la cual se sugiere bienal después de los 50 años ${ }^{18}$. Entretanto en las mujeres norteamericanas, se sugiere ésta última cada dos años después de los 40 años de edad ${ }^{10,11}$.

El uso de los servicios de salud es una conducta dinámica de la población en donde su capacidad de interpretación juega un papel muy importante ${ }^{19}$. Se han descrito diferentes modelos que explican este fenómeno, entre ellos psicosocial ${ }^{20,21}$, que describe los supuestos por los que una persona decide efectuarse alguna detección o prueba con fines preventivos. Para ello, se necesita tener una disposición para tomar medidas respecto a determinada condición de salud; la persona cree o está convencida de que el 
someterse a esa acción preventiva reduce el riesgo con respecto a la enfermedad o a la gravedad de ésta. Existe además la presencia de un estímulo para realizar la acción. Estas situaciones hacen posible la utilización de los servicios de salud. Así, en el proceso de decisión de realizar acciones respecto a la salud de una persona interviene el grado en que está dispuesta a actuar, sus creencias y las que determina la sociedad sobre la eficacia de medidas alternativas; aunado a barreras psicológicas, influencias interpersonales y una o más señales que desencadenan una respuesta.

Se ha mencionado que para que la población utilice los servicios preventivos, es necesario que se reduzcan las barreras financieras $^{22,23}$, se mejore el acceso geográfico y se haga conciencia de la importancia de obtener servicios preventivos de salud ${ }^{22}$. Berraondo y cols ${ }^{24}$ reportaron que el 79,3\% de una población española usó la detección de hipertensión en un 79,3\% en los últimos dos años, en tanto que el $32,2 \%$ de las mujeres se realizó la mamografía en alguna ocasión; y el 43,7\%, la citología cervical en los últimos dos años. Por otra parte, una población de nativos americanos observó un alto consumo de servicios preventivos, ejemplo de ello es la detección de hipertensión que durante el año anterior se la había practicado un $85 \%$; en tanto que el $63,3 \%$ y $71,7 \%$ de las mujeres se practicaron durante el año anterior, una exploración física de mamas y una citología cervical, respectivamente ${ }^{25}$.

En México, el uso de las acciones preventivas es conocido por los reportes que hacen las instituciones que conforman el SSM, los cuales han notificado para el año 2000 una cobertura de $24,6 \%$ y $41,6 \%$ en DDM y DHTA, respectivamente ${ }^{26}$; y $57,8 \%$, en $\mathrm{DCACU}^{4}$. Por otra parte, la última Encuesta Nacional de Salud, informó que la población mayor de 20 años usa los servicios preventivos en el último año un 10,5\% y 13,4\% para DDM y DHTA, respectivamente; para DCACU, un $27,4 \%$; y para DCAMA, menos del $10,0 \%$ de las mujeres del mismo grupo de $\operatorname{edad}^{27}$.

Monterrey y su zona metropolitana se encuentra situada en el Estado de Nuevo León al nordeste de México, incluye ciudades cuya población se dedica principalmente a las actividades de la industria manufacturera, el comercio y la construcción. El 39,5\% de los habitantes son económicamente activos, de los cuales el $77,1 \%$, es empleado u obrero. El 62,1\% es usuario de la seguridad social (población que hace uso de los servicios institucionales por lo menos una vez en el año) ${ }^{28}$.

El SSM se conforma por instituciones de seguridad social, de asistencia social y servicios privados, y en cada uno de éstos se da atención tanto en los programas de prevención de enfermedades como en los de asistencia médica. Así, el SSM implementa diferentes programas de prevención, observándose por una parte en los informes institucionales el uso de acciones preventivas, lo cual facilita la planeación de recursos destinados a la salud. Por otra parte, en encuestas nacionales, donde el resultado es proporcionado en forma general y detallado para los diferentes estados que integran el país. Aún así, no todos los datos se encuentran disponibles para cada lugar. Además es necesario realizar acciones específicas encaminadas a lograr el máximo de eficiencia en el uso de los recursos tanto en las instituciones como en la población. Lo anterior hace patente la necesidad de determinar con base poblacional de Monterrey y su zona metropolitana, la utilización de DDM, DHTA, DCACU, DCAMA y toxoide tetánico diftérico (TTD).

\section{SUJETOS Y MÉTODOS}

La población de estudio consistió en personas 25 años y más, habitantes de Monterrey, México y nueve municipios que conforman su área metropolitana. Se excluyeron a los que padecían diabetes mellitus, hiper- 
tensión arterial, cáncer cérvicouterino y cáncer mamario. El tamaño de la muestra $(\mathrm{n}=$ 254) se calculó con base al supuesto de que un 20 por ciento o menos de la población usó alguna acción preventiva en el último año, mediante la fórmula para estimar una proporción en una población finita con un error esperado del 5 por ciento y un intervalo de confianza, del 95 por ciento.

La distribución de la muestra se hizo proporcional a la población de 25 años y más de cada municipio del área metropolitana de Monterrey ${ }^{29}$. Se seleccionaron al azar simple tres Áreas Geoestadísticas Básicas (AGEB's) que son unidades integradas por un conjunto de manzanas edificadas y delimitadas por calles y avenidas ${ }^{30}$, cuando se requerían más de 39 personas por municipio; y un AGEB, en caso de menos personas. Se eligieron proporcionalmente y al azar una, dos y cuatro manzanas en cada AGEB. Los domicilios se asignaron en forma sistemática; primero se contó el total de domicilios habitados, después se calculó el número necesario de los mismos (No. domicilios en la manzana / No. domicilios requeridos). Finalmente, en caso de encontrarse en el domicilio seleccionado más de una persona con las características requeridas para participar en el estudio, se eligió una de ellas para colaborar en el estudio usando la técnica aleatoria simple. Si la persona seleccionada se rehusaba a participar y en el domicilio hubiera más personas, de éstas se seleccionaba otra; solo si ninguna hubiese querido participar se ocurría al domicilio siguiente. Se procedió de la misma manera para suplir a las personas que no se encontraban en el domicilio; en total se reemplazaron doce personas. La información fue reunida con la ayuda de cinco encuestadores que fueron previamente capacitados, éstos contaban con estudios técnicos y experiencia en trabajo comunitario. Los datos se colectaron en forma de encuesta en el domicilio de las personas, a los que se acudió en diferentes días incluyendo sábados y domingos, también en distintos horarios para evitar el tener solo a un grupo de la población en los domicilios.

Para valorar el uso de las acciones preventivas, se consideró a la población que se realizó en el último año, DDM mediante glucemia capilar; DHTA, a través de una toma de presión arterial; DCAMA, con base a exploración física de mamas; DCACU, mediante citología cérvicouterina; y TTD, a través de aplicación de por lo menos una dosis. Además se colectaron las siguientes variables sociodemográficas: sexo (femenino, masculino); edad (años cumplidos [ $\leq 64$ años y $\geq 65$ años]); estado civil (con pareja [casado o en unión libre] y sin pareja [soltero, viudo o divorciado]); escolaridad (ninguna y educación elemental, educación media y media superior, y superior); ocupación (ama de casa, empleado, desempleado y pensionado o jubilado); tipo de servicio médico (seguridad social, asistencia social y servicios médicos privados); y lugar donde se efectuó las acciones preventivas (seguridad social, asistencia social y servicios médicos privados). Se preguntó también acerca del antecedente familiar directo de diabetes mellitus; hipertensión arterial; cáncer cérvicouterino; y cáncer mamario. Asimismo, se interrogó sobre asistencia a sesiones de educación para la salud durante el año anterior e importancia que para la persona tiene el efectuarse detecciones, inmunizaciones y asistir a sesiones de educación para la salud (nada de importancia, poco importancia, regular importancia, importante y muy importante). Finalmente, se indagó acerca de quién le envió a efectuarse sus acciones preventivas (yo solo, el médico, la enfermera y otro personal de salud).

El análisis estadístico consistió en estadística descriptiva, calculando promedios, desviaciones estándar, porcentajes y sus intervalos de confianza del 95 por ciento; se realizó análisis bivariado con las variables de percepción de importancia y antecedentes familiares de diabetes mellitus, hipertensión arterial, cáncer cérvicouterino y cáncer mamario. 


\section{RESULTADOS}

La edad promedio de la población estudiada fue de 42,3 años $\pm 14,0$ años, el $65,4 \%$ (166) correspondió al sexo femenino y un $76,6 \%$ (193), contaba con pareja. El 42,9\% (109) presentaba escolaridad elemental o no tenía ningún tipo de estudios. En tanto que el $51,8 \%$ (130), se dedicaba a las actividades del hogar. Un 74,8\% (190) de las personas refirió tener seguridad social mientras que un 24,4\% (62), asistencia social; el resto comentó asistir a la atención privada. Al responder acerca del antecedente familiar en un consanguíneo directo, un 37,0\% (94) dijo tener de diabetes mellitus y un 39,0\% (99), de hipertensión arterial; las mujeres reconocieron contar con antecedente de cáncer cérvicouterino y cáncer mamario un 1,8\% (3) y $3,0 \%$ (5), respectivamente.

El 16,5\% (42) mencionó haber asistido durante el último año a sesiones de educación para la salud. Al responder acerca de la percepción de importancia de las diferentes actividades que se llevan a cabo en medicina preventiva, un 50,8\% (129) de las personas respondió que era muy importante efectuar acciones preventivas en lugar de curar o tratar de solucionar los problemas de salud cuando estos aparecen. Mientras que un 51,2\% (130) opinó de la misma manera al responder acerca de la importancia de asistir a efectuarse detecciones. Un 54,7\% (139) opinó que era muy importante asistir a aplicarse las inmunizaciones correspondientes, el 40,9\% (104) respondió que es muy importante asistir a sesiones educativas para la salud.

En lo referente al uso de acciones preventivas, el 53,5\% (136) reconoció haber utilizado una o más de ellas en el último año; 98,5\% (134), se las realizó en instituciones de seguridad social y $1,5 \%$ (2), en instituciones de asistencia social. Un 41,2\% (56) acudió espontáneamente a solicitar el servicio, mientras que un 44,1\% (60) fue enviado por su médico; el resto, fue enviado por diferente personal de salud tanto de las unidades médicas como de sus lugares de trabajo.

En lo que respecta a la utilización de las acciones preventivas, un $37,0 \%$ (94) (IC $95 \% 31,43)$ mencionó haberse realizado

Figura 1

Utilización de acciones preventivas por 254 personas de Monterrey (México) y su zona metropolitana, por sexo

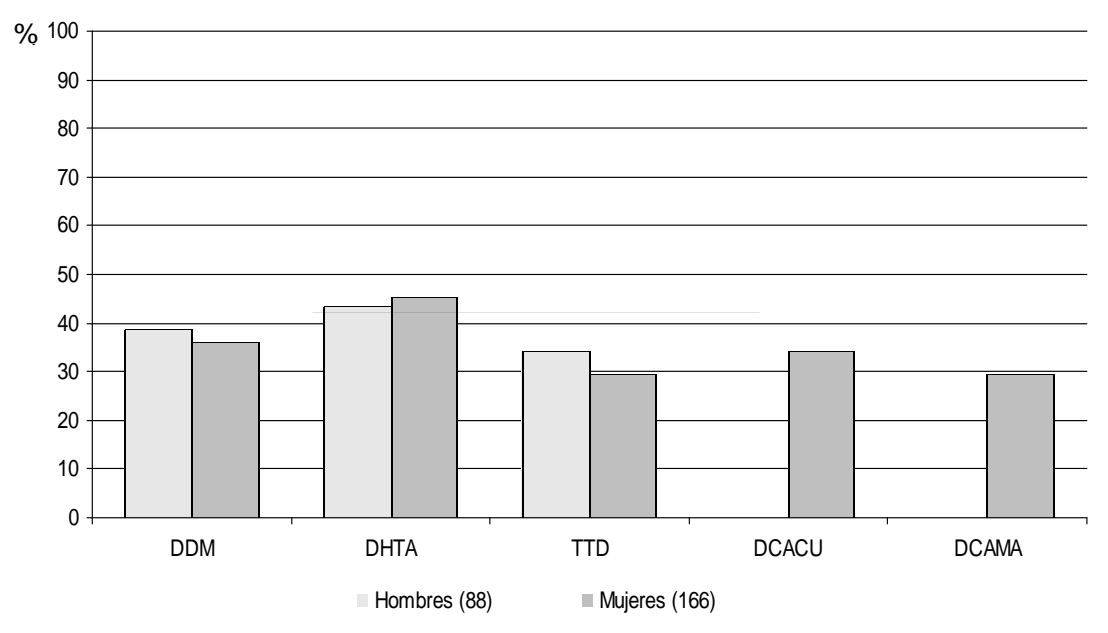

DDM: Detección de diabetes mellitus, DHTA: Detección de hipertensión arterial, TTD: Toxoide tetánico diftérico, DCACU: Detección de cáncer cérvico uterino, DCAMA: Detección de cáncer mamario. 
Figura 2

Utilización de acciones preventivas por 254 personas de Monterrey (México) y su zona metropolitana, por edad

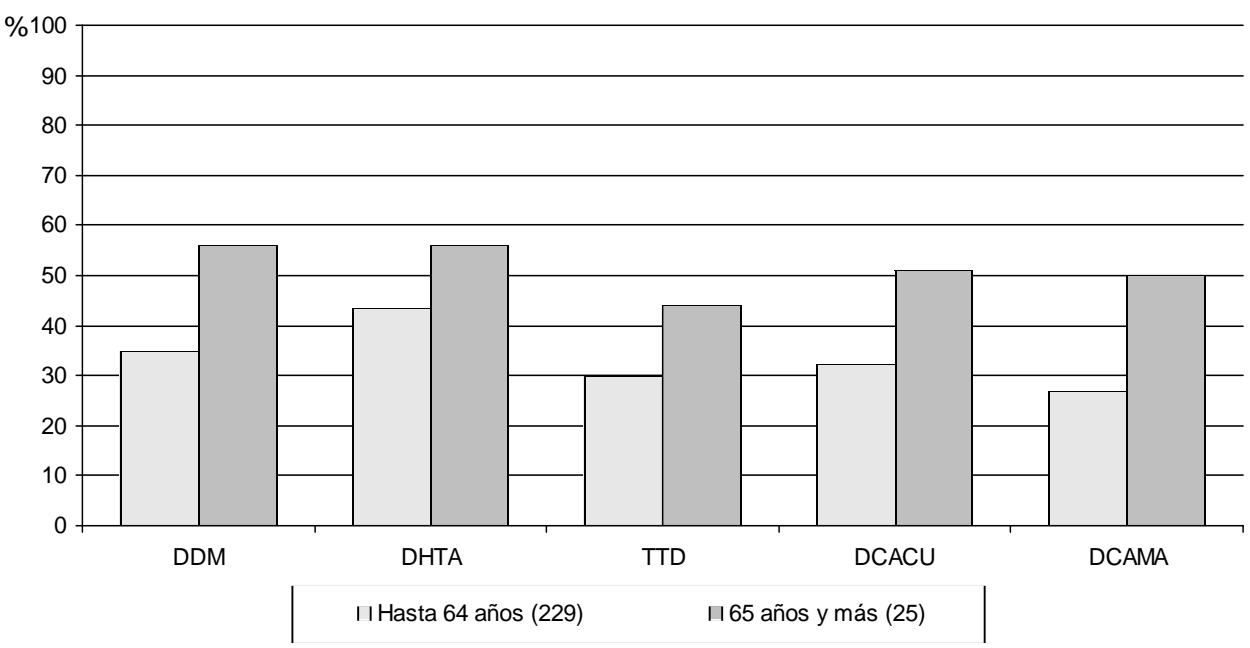

DDM: Detección de diabetes mellitus, DHTA: Detección de hipertensión arterial, TTD: Toxoide tetánico diftérico, DCACU: Detección de cáncer cérvico uterino, DCAMA: Detección de cáncer mamario.

DDM; el 44,5\% (113) (IC 95\% 38, 51), DHTA; y el 31,1\% (79) (IC 95\% 27, 37), TTD. En las detecciones propias de la mujer, un 34,3\% (57) (IC 95\% 27, 42) de la población femenina se efectuó DCACU durante el año anterior, mientras que un $29,5 \%$ (49) (IC $95 \%$ 23, 36), DCAMA.

Al analizar el comportamiento de la utilización de las acciones preventivas estratificando por las variables sociodemográficas se observó para el caso de sexo, que la utilización de DDM y DHTA varió de un 36,1\% a un $45,2 \%$ (Figura 1); mientras que en el caso del grupo de edad de 65 años, todas las detecciones se usaron por un 50,0\% o más de la población (figura 2). En el cuadro I se observa la utilización de las acciones preventivas con el resto de las variables sociodemográficas. No se encontró relación de las variables antecedente familiar y percepción de importancia con el uso de las acciones preventivas, tampoco con las variables sociodemográficas.

\section{DISCUSIÓN}

En el marco legal mexicano, todos tienen derecho a la salud. Así, en el Estado de Nuevo León como se mencionó anteriormente, más de la mitad de la población es usuaria del régimen de la seguridad social, mientras que el resto es atendido tanto en área asistencial como de salud pública en instituciones privadas y de asistencia social. Situación similar a la encontrada en la población estudiada, ya que tres cuartas partes de ella contaban con algún régimen de seguridad social.

Se observó que la mayor parte de los encuestados realizó sus detecciones e inmunizaciones en instituciones de seguridad social, situación que pudiera ser explicada debido a que estos programas de prevención están considerados como prioritarios en el Sector Salud ${ }^{4-8}$, lo que hace posible la realización de estas acciones en dichas instalaciones, aún sin la correspondiente afiliación. También pudiera influir la disponibilidad de 
Tabla 1

Utilización de acciones preventivas por 254 personas de Monterrey (México) y su zona metropolitana

\begin{tabular}{|c|c|c|c|c|c|}
\hline & $\begin{array}{c}\text { DDM* } \\
\% \\
\text { (frecuencia) }\end{array}$ & $\begin{array}{c}\text { DHTA* } \\
\% \\
\text { (frecuencia) }\end{array}$ & $\begin{array}{c}\text { TTD* } \\
\% \\
\text { (frecuencia) }\end{array}$ & $\begin{array}{c}\text { DCACU* } \\
\% \\
\text { (frecuencia) }\end{array}$ & $\begin{array}{c}\text { DCAMA* } \\
\% \\
\text { (frecuencia) }\end{array}$ \\
\hline $\begin{array}{l}\text { Estado civil } \\
\text { Con pareja }(n=193) \\
\text { Sin pareja }(n=59)\end{array}$ & $\begin{array}{l}37,8(73) \\
33,9(20)\end{array}$ & $\begin{array}{l}45,6(88) \\
40,7(24)\end{array}$ & $\begin{array}{l}31,6(61) \\
28,8(17)\end{array}$ & $\begin{array}{l}35,1(46) \\
32,4(11)\end{array}$ & $\begin{array}{c}30,5(40) \\
26,5(9)\end{array}$ \\
\hline $\begin{array}{l}\text { Ocupación } \\
\text { Hogar }(\mathrm{n}=130) \\
\text { Empleado }(\mathrm{n}=107) \\
\text { Jubilado/ Pensionado }(\mathrm{n}=12)\end{array}$ & $\begin{array}{c}37,7(49) \\
33,6(36) \\
58,3(7)\end{array}$ & $\begin{array}{c}49,2(64) \\
39,3(42) \\
58,3(7)\end{array}$ & $\begin{array}{l}30,8(40) \\
31,8(34) \\
33,3(4)\end{array}$ & $\begin{array}{l}36,2(47) \\
27,3(9) \\
50,0(1)\end{array}$ & $\begin{array}{c}32,3(42) \\
21,2(7) \\
0,0(0)\end{array}$ \\
\hline $\begin{array}{l}\text { Escolaridad } \\
\text { Elemental }(\mathrm{n}=109) \\
\text { Media }(\mathrm{n}=116) \\
\text { Superior }(\mathrm{n}=29)\end{array}$ & $\begin{array}{c}40,4(44) \\
38,8(45) \\
17,2(5)\end{array}$ & $\begin{array}{c}49,5(54) \\
44,0(51) \\
27,6(8)\end{array}$ & $\begin{array}{c}29,4(32) \\
33,6(39) \\
27,6(8)\end{array}$ & $\begin{array}{c}38,3(31) \\
31,9(22) \\
25,0(4)\end{array}$ & $\begin{array}{c}28,4(23) \\
31,9(22) \\
25,0(4)\end{array}$ \\
\hline $\begin{array}{l}\text { Servicios de salud } \\
\text { Asistencia social }(\mathrm{n}=62) \\
\text { Seguridad social }(\mathrm{n}=190) \\
\text { Servicios privados }(\mathrm{n}=2)\end{array}$ & $\begin{array}{l}19,4(12) \\
42,1(80) \\
100,0(2)\end{array}$ & $\begin{array}{c}17,7(11) \\
53,2(101) \\
50,0(1)\end{array}$ & $\begin{array}{c}11,3(7) \\
37,9(72) \\
0(0)\end{array}$ & $\begin{array}{c}9,7(3) \\
40,0(54) \\
0(0)\end{array}$ & $\begin{array}{c}6,5(2) \\
34,8(47) \\
0(0)\end{array}$ \\
\hline
\end{tabular}

DDM: Detección de diabetes mellitus, DHTA: Detección de hipertensión arterial, TTD: Toxoide tetánico diftérico, DCACU: Detección de cáncer cérvico uterino, DCAMA: Detección de cáncer mamario.

recursos de las instituciones o bien, las barreras de acceso ${ }^{22,23}$, situación que deberá de ser estudiada posteriormente.

Así, a pesar de que en todas las instituciones de Sector Salud se puede hacer uso de las acciones preventivas, poco más de la tercera parte de la población se realizó toma de muestra de glucemia capilar. En el ámbito nacional se informó que en el año 2000 se realizaron 11 millones de detecciones, es decir, un $20,8 \%$ con relación a la población de 20 años y más en el mismo año ${ }^{8,28}$. Esta situación se torna como un foco de alerta ya que por una parte un alto porcentaje de los habitantes tiene afiliación a la seguridad social y por la otra, todos tienen fácil acceso a cualquier institución de salud, por ser un programa preventivo prioritario. Además, conocida es la relevancia de los beneficios de esta detección, ya que el identificar oportunamente una enfermedad crónica como la diabetes, tratarla oportuna y adecuadamente, retarda la aparición de sus complicaciones ${ }^{8}$.

El uso de DHTA es menor a lo mencionado en población española de cinco municipios con población urbana y rural, todos adscritos a un centro de salud donde el 79,3\%, refirió haberse realizado una toma de presión arterial en los últimos dos años ${ }^{24}$. Mientras que en la evaluación efectuada al pro- 
grama de actividades preventivas y de promoción a la salud (PAPPS) se reportó que para 1999, en personas adscritas a centros de salud españoles el 43,0\% de la población contaba con esta actividad preventiva en forma correcta ${ }^{31}$. Es necesario entonces, que se tomen medidas al respecto, ya que se ha documentado que la hipertensión se presenta con una prevalencia de riesgo para enfermedades cardiovasculares de $30,05 \%$ en mayores de 20 años ${ }^{7}$. La situación se torna reveladora ya que es imprescindible que las instituciones que se encargan de promover la salud realicen su máximo esfuerzo en la búsqueda intencionada de personas con factores de riesgo.

Respecto al uso de TTD, poco más de la cuarta parte de la población refirió su aplicación, muy parecido a lo reportado en población española donde el 39,1\% contaba con el esquema correcto de la vacunación antitetánica $^{31}$. Sin embargo, en este trabajo no se revisó el uso adecuado del esquema de aplicación, es decir, sólo se cuestionó si había recibido el toxoide el año anterior, por lo que algunos entrevistados podrían haber tenido una aplicación vigente de la inmunización y por lo tanto no estaban en la necesidad de contar con la misma. Cabe mencionar que actualmente en el esquema de vacunación para adultos se encuentran además, la vacuna doble viral (sarampión, rubéola), la antiinfluenza y la antineumocócica todas ellas dirigidas a grupos específicos de población ${ }^{14-16}$. Estas últimas inmunizaciones no fueron indagadas en este trabajo debido a que el TTD es más conocido para la población que el resto de las vacunas y para no producir confusión en las personas encuestadas. Estos esquemas de vacunación se incluyen en los estándares básicos de vacunación para adultos en población de Estados Uni$\operatorname{dos}^{10}$.

En lo referente a las detecciones propias de la mujer, se ha informado que mujeres con diferentes planes de salud se realizan la DCACU en un $74 \%, 65 \%$ y $53 \%$, de acuerdo a los planes de pago correspondientes ${ }^{31}$. Por otra parte, se ha dicho que mujeres de 18 años o más, sin histerectomía y que residen en área urbana, rural cercana y rural más alejada, reportaron en la encuesta telefónica del Sistema de Vigilancia de Factores de Riesgo de Conducta de 1997, la práctica de esta detección en los pasados tres años en un $85,9 \%, 82,4 \%$ y $81,4 \%$, respectivamente ${ }^{22}$. En tanto, un $43,7 \%$ de mujeres españolas adscritas a un centro de salud mencionaron en encuesta telefónica, haberse efectuado el estudio en los últimos dos años ${ }^{24}$, mientras que Subías y cols. ${ }^{31}$ documentaron el uso correcto por el $20,1 \%$ de la población y en el caso del presente trabajo sólo tres de cada diez mujeres, se realizaron la DCACU. Vale la pena comentar este punto, el cáncer cérvicouterino es una enfermedad que detectada en sus estadios tempranos, permite llevar tratamientos oportunos para lograr evitar la evolución a etapas avanzadas ${ }^{4}$. Es pertinente que se implementen estrategias para el logro de este objetivo, ya se ha dicho que hay incremento de las tasas de detecciones de cáncer cuando se realizan cambios en la organización tales como uso de visitas programadas para prevención y asignación de equipo no médico para actividades preventivas, así como de involucrar a los pacientes mediante incentivos financieros y avisos recordatorios, lo que hace posible la realización de cambios favorables ${ }^{32}$.

Berraondo y cols. ${ }^{24}$ reportaron que un $56,8 \%$ de mujeres españolas se realizaron una mamografía en los últimos dos años, también en España, Subías y cols. ${ }^{31}$ reportaron que un $12,3 \%$ y $6,6 \%$ contaban con mamografía y exploración mamaria respectivamente. Mientras que en población urbana de Estados Unidos se la realizaron en los últimos dos años un 67,6\%; en el área rural, un 59,3\%; y en las más alejadas, un $59,4 \%{ }^{22}$. Así, en tanto que en otros países hacen uso de un recurso diagnóstico como lo es la mamografía, en este trabajo solo se indagó acerca de la exploración física de las mamas por personal de salud. Pero como ya se ha 
comentado, la mamografía es un recurso que se usa en población mayor de 40 años y con presencia de otros factores de riesgo ${ }^{17}$.

Es sabido que en México se ha incrementado la esperanza de vida, en el año 2000 el $5 \%$ de la población era de 65 años o más. Este grupo, utiliza los servicios de salud cuatro veces más que el resto de la población ${ }^{3}$, por lo que es necesario que las acciones preventivas sean un aspecto importante en ellos. En la población estudiada se observó que este grupo de personas usó en mayor proporción las actividades preventivas, situación que debiera ser analizada posteriormente, ya que este no fue uno de los fines de este trabajo. Por otra parte el comportamiento que se observó del uso de acciones preventivas por sexo es superior a lo reportado en la Encuesta Nacional de Salud que investigó acerca del uso de acciones preventivas en el año anterior a la misma ${ }^{27}$. Esto pudiera ser explicado por el hecho de que el lugar donde se realizó el estudio es una zona industrial y ambos sexos juegan un papel muy importante en la actividad económica de la zona, contando además con los beneficios de la seguridad social, brigadas médicas y puestos de fábrica por parte de las empresas en las que laboran.

Si bien, las proporciones de uso encontradas en la población estudiada están por debajo de las reportadas en la literatura, es pertinente señalar que en este trabajo se indagó solo acerca del comportamiento en el año anterior, no del uso correcto de las detecciones e inmunizaciones para adultos, situación que pudiera explicar la baja utilización de éstas y que debiera ser estudiada en futuras investigaciones para evaluar este tipo de programas. Además, es pertinente señalar que en el estudio no se hizo revisión de expedientes clínicos o tarjetas de programas de las instituciones de salud, en donde se registran las acciones médico preventivas que se efectúan al paciente; el dato se obtuvo directamente del interrogatorio al paciente, lo que habría que tomar en cuenta ya que podría influir el olvido de la persona o también el desconocer las acciones que se le realizan.

Es importante mencionar que mientras en otros países los programas preventivos incluyen en sus estándares mínimos otras acciones preventivas ${ }^{10,11}$, en México, se ha propuesto la detección integral para la diabetes mellitus, hipertensión arterial y obesidad que permite un mejor control metabólico, además de evitar o retardar las complicaciones $^{7,8}$. Esta situación propicia una línea de investigación referente a los recursos destinados a la salud, ya que en la práctica se envían a estudios de diagnóstico solo a aquellas personas en las que se les detecta algún signo de alarma.

Es necesaria la búsqueda de los factores que son detonantes para que la población use las acciones preventivas, en esta población fueron mínimas las personas que tuvieron antecedentes familiares con diabetes mellitus, hipertensión arterial, cáncer cérvicouterino y cáncer mamario. La gran mayoría percibía que era de importante a muy importante efectuarse este tipo de acciones, pero no hubo relación de estas variables con el uso. Un inconveniente a esto podría ser la falta de muestra para este tipo de planteamiento, por lo que se propone seguir con esta línea de investigación ya que es un fenómeno que ha sido estudiado con anterioridad ${ }^{20}$.

Sí bien, el uso de acciones preventivas por parte de la población es un evento que puede ser reportado con los informes de actividades de las unidades de salud, es importante destacar que este proyecto se realizó en población abierta. Situación que toma especial interés ya que incluyó a diez municipios, cuyas características geográficas hacen posible que en éstos se concentre el $80 \%$ de la población estatal ${ }^{28}$. Estudios como este apoyan de gran manera las políticas de salud en el país, ayudando a localizar focos de alerta en los diferentes programas preventivos en donde se tengan que implementar estrategias como la búsqueda intencionada 
de personas enfatizando en estilos de vida y cambios de conducta, tratando de reducir así el riesgo y logrando con ello la salud de la población.

\section{BIBLIOGRAFÍA}

1. Frenk J; La Salud de la Población, Hacia una Nueva Salud Pública. México: Fondo de Cultura Económica; 1994.

2. Secretaría de Salud. Estadísticas de morbimortalidad. México: Secretaría de Salud; 2001.

3. Secretaría de Salud. Programa Nacional de Salud 2001-2006. México: Secretaría de Salud; 2001.

4. Secretaría de Salud. Programa de acción: Cáncer cérvico uterino. México: Secretaría de Salud; 2002.

5. Secretaría de Salud. Programa Estatal de Salud 1997-2003. México: Subsecretaría de Salud. Estado de Nuevo León; 1997.

6. Secretaría de Salud. Programa de acción: Cáncer de mama. México: Secretaría de Salud; 2002.

7. Secretaría de Salud. Programa de acción: enfermedades cardiovasculares e hipertensión arterial. México: Secretaría de Salud; 2001.

8. Secretaría de Salud. Programa de acción: diabetes mellitus. México: Secretaría de Salud; 2001.

9. Secretaría de Salud. NOM-015-SSA2-1994.Modificación a la Norma Oficial mexicana NOM-015SSA2-1994. Para la prevención, tratamiento y control de la diabetes mellitus en la atención primaria para quedar como Norma Oficial Mexicana NOM015-SSA2-1994, Para la prevención, tratamiento y control de la diabetes. México: Secretaría de Salud; 1994.

10. Put prevention into practice. A sep by sep guide to delivering clinical preventive services: a systems approach. USA: Agency for health care research and quality; 2002. Disponible en: .

11. Guide to clinical preventive services, Third Edition: Periodic Updates. AHRQ Publication No. 030007, March 2003. Agency for Health Care Research and Quality, Rockille, MD. Disponible en: http://www.ahrq.gov./clinic/periodorder.htm.

12. Secretaría de Salud. NOM-030-SSA2-1999. Norma Oficial Mexicana NOM-030-SSA2-1999. Para la prevención, tratamiento y control de la hipertensión arterial. México: Secretaría de Salud; 1999.

13. Villar Álvarez F, Maiquez galán A, Brotons Cuixart C, Trocal Laguna J, Lorenzo Piqueres A, Vilaseca Canals J, Banegas Banegas JR. Prevención cardiovascular en atención primaria. Aten Primaria 2001; 28 (supl 2): 13-26.

14. Instituto Mexicano del Seguro Social. Programas integrados de salud. Salud de la mujer de 20 a 59 años. México: Instituto Mexicano del Seguro Social. 2002.

15. Instituto Mexicano del Seguro Social. Programas integrados de salud. Salud de los hombres de 20 a 59 años. México: Instituto Mexicano del Seguro Social. 2002.

16. Instituto Mexicano del Seguro Social. Programas integrados de salud. Salud de los adultos mayores de 59 años. México: Instituto Mexicano del Seguro Social. 2002.

17. Secretaría de Salud. NOM-014-SSA2-1994. Modificación a la Norma Oficial Mexicana NOM-014SSA2-1994, Para la prevención, tratamiento y control del cáncer del cuello de útero y mamario en la atención primaria, para quedar como NOM-014SSA2, Para la prevención, detección, diagnóstico, tratamiento, control y vigilancia epidemiológica del cáncer cérvico uterino. México: Secretaría de Salud; 1994.

18. Bellas Beceiro B, Cierco Peguera P, González Enríquez J, Martín Blanco N, Melús Palazón E, Alonso Gordo JM, Marzo Castillejo M. Prevención del cáncer. Aten Primaria 2001; 28 (supl 2): 52-81.

19. Bronfman M, Castro R, Zúñiga E, Miranda C, Oviedo J. Del "cuánto" al "por qué": la utilización de los servicios de salud desde la perspectiva de los usuarios. Salud Pública de México 1997; 39: 44250.

20. Rosenstock I. Por qué la gente utiliza los servicios de salud. En: White K, Frenk J, Ordóñez C, Paganini JM, Starfield B, editores. Investigaciones sobre servicios de salud: una antología. Organización Panamericana de la Salud, 1992.p. 405-24.

21. Arredondo A; Meléndez V. Modelos explicativos sobre la utilización de servicios de salud: revisión y análisis. Salud Pública de México 1992; 34: 36-49.

22. Casey MM, Thiede K, Klingner JM. Are rural residents less likely to obtain recommended preventive healthcare services. Am J Prev Med 2001; 21: 18288.

Rev Esp Salud Pública 2004, Vol. 78, N. ${ }^{\circ} 1$ 
23. Schillinger JA, Craig BA, Austin D, Jack L, Heumann M, Moore J, et al . Health care reform in Oregon: The impact of the Oregon Health Plan on utilization of mammography. Am J Prev Med 2000; 18: 11-7.

24. Berraondo I, Alkiza ME, Santacoloma I, Calvo A, Tapiz V, Bergera A, Martínez JM. Conocimiento de la utilización de prácticas preventivas a través de la encuesta telefónica. Atención Primaria 1996; 18: 131- 5 .

25. Gilliland F, Mahler R, Hunt C, Davis S. Preventive health care among American Indians in New Mexico. Preventive Medicine 1999; 28: 194-202.

26. Secretaría de Salud. Metas del Programa Nacional de Salud 2001 - 2006. México: Secretaría de Salud; 2001.

27. Oláiz G, Rojas R, Barquera S, Shamah T, Cravioto P, López P, et al. Encuesta Nacional de Salud 2000. Tomo 2. La salud de los adultos. México: Instituto Nacional de Salud Pública; 2003.

28. Instituto Nacional de Estadística Geografía e Informática. Anuario Estadístico, Nuevo León. México:
Instituto Nacional de Estadística Geografía e Informática; 2001.

29. Instituto Nacional de Estadística Geografía e Informática. IX Censo Nacional de población y vivienda. México: Instituto Nacional de Estadística, Geografía e Informática. 1990.

30. Instituto Nacional de Estadística Geografía e Informática. Perfil sociodemográfico. México: Instituto Nacional de Estadística Geografía e Informática; 1997.

31. Subías Lorén PJ, Bauzá Nicolay K, Casanovas Cuquet E, García Mata JR, Iglesias Rodal M, Jiménez Villa J, et. al. Evaluación del PAPPS. Aten Primaria 2001; 28 (supl 2): 15-21.

32. Weisman C, Hederson J. Managed care and women's care, preventive services, and satisfaction. Women's Health Issues 2001; 11: 201-215.

33. Stone EG, Morton SC, Hulscher ME, Maglione MA, Roth EA, Grimshaw JM, et al. Interventions that increase use of adult immunization and cancer screening services: a meta-analysis. Ann Intern Med 2002; 136: 641-51. 